\title{
A Network-Based Management Information System for Animal Husbandry in Farms
}

\author{
Jing $\operatorname{Han}^{1}$ and Xi Wang ${ }^{2, *}$ \\ ${ }^{1}$ College of Information Technology, Heilongjiang August First Land Reclamation University, \\ Daqing, Heilongjiang Province, P.R. China 163319 \\ ${ }^{2}$ College of Engineering, Heilongjiang August First Land Reclamation University, \\ Daqing, Heilongjiang Province, P.R. China 163319, \\ Tel.: +86-459-6819224; Fax: +86-459-6819224 \\ hanj1202@163.com, ndwangxi@163.com
}

\begin{abstract}
It has already been a trend for the management of animal husbandry in farms to employ the advanced management system(MIS)and the softwaredeveloping platform to scientifically manage the information about animal husbandry in farms through the network. This is a network system that combines $\mathrm{B} / \mathrm{S}$ structure and ASP techniques, and the method adopts both the computer network technology and database technology to implement system and data integration. Based on the characteristics of the system, the security of the management information system is studied. The security strategy model of the MIS is proposed, and the technical strategy of the MIS's security is discussed in the level of the application software and the database. And the security management method of the $\mathrm{B} / \mathrm{S}$ structure is introduced briefly.
\end{abstract}

Keywords: management information system, ASP, animal husbandry information, network technology.

Currently, the economy of agriculture and rural areas of China is now developed to a new historical phase, and animal husbandry has gradually evolved into the preponderant and predominant industry in the economic development of rural areas (Kou Zhanying). In the agricultural production of the reclamation areas in Heilongjiang Province, animal husbandry accounts for a significant proportion. The problems of traditional method of animal husbandry information management have inevitably come up, such as inadequate in information, low in efficiency and slow in feedback. The informationization of animal husbandry is a way to spur the modernization of animal husbandry through informationization, combining information technology, network technology, artificial intelligence technology, computer technology and modern management theory. And this will have far-reaching significance to promoting the economy of rural areas and agricultural development. Establishing a management information system of animal husbandry (MISAH) is beneficial to raising the efficiency of information work in animal husbandry and quickens the progress of animal husbandry informationization. And setting up such a system in Heilongjiang Province is

\footnotetext{
* Corresponding author.
} 
also conducive to increasing the degree of local husbandry information management, and making it more normalized and routinized, thus providing reliable foundation for the informationization of animal husbandry in the overall reclamation areas. Building up such a system can make full use of the resource of animal husbandry in Heilongjiang province, steering it into creating greater value for production of animal husbandry, and it can also meet the needs of developing modern animal husbandry, thus exerting considerable influence on the economic development of the country.

\section{The Analysis of the System and Overall Design}

\subsection{The Concept of the Design of the System}

The system has as its developing object the Red Star farm in the reclamation area of Heilongjiang province. According to its functions, the management information system (MIS) can be divided into four comparatively independent modules. For the individual module, its needs are easy to realize. Therefore, based on the above-mentioned analysis and comparison and based on the realistic situation of the Red Star Farm, it's decided that the development of the system will utilize the method of prototype and that of structural life period. The former analyzes the needs, with the modified and identified prototype system as the basis for developing the system, and the latter is used to design. Standard datasheets are adopted in the process of system-designing, the transmission of data will be realized through the system of data exchange, and the standard of statistics in the datasheets will be normalized, to ensure the normalization of the data and the safety of transmission.

The reclamation area in Heilongjiang province is vast in its extent, and naturally the economic situations and natural environments are diverse, so the information system platforms of animal husbandry to be developed should have different focus. The system is designed according to the characteristics of the Red Star Farm, and the following principles are followed in designing and developing the integrated platform of MISAH.

(1) The commonality should be taken into account in designing the system platform, and its content should be combined with the characteristics of the local place, giving prominence to its direction, thus making it convenient to use for the animal husbandry department and people engaged in animal husbandry.

(2)The operation of the project should be simplified, functionalized, giving full play to the role of information system platform.

(3) The system can analyze, sort out and mobilize the information on animal husbandry in the Red Star Farm.

(4)The system upholds the graphical user interface designing principle, the interface is direct-viewing and transparent to users.

(5)When users use the software, they can fully understand the functions of the interface, and without much training they can conveniently use the system.

The system can be applicable to the other farms with simple modification.

\subsection{The Analysis of the Functions of the System}

According to investigation, analysis and categorization of the needs of MISAH, it can be divided into the subsystems of animal husbandry production management, of 


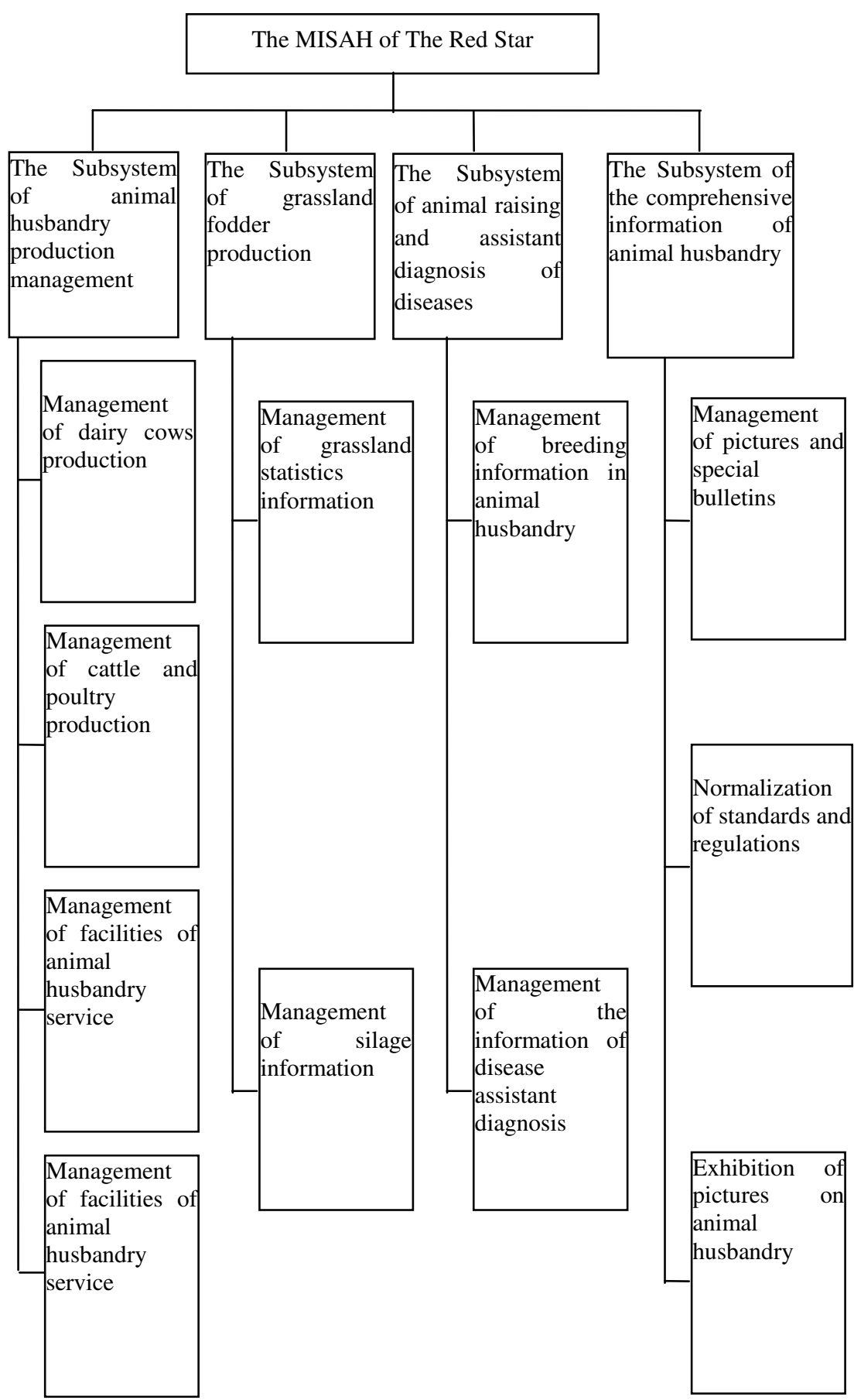

Fig. 1. Main structure of functional module 
grassland fodder production, of the animal-raising and the assistant diagnosis of diseases, and of the comprehensive information of animal husbandry, with every subsystem falling into several modules. Thus, such functions can be realized such as information input, search, modification, back-up, collection, data analysis and information classification. Its diagram is shown in Fig.1.

The system designate three different roles to various users: system administrators, the administrators of subsystems (the personnel in animal husbandry administration of farms), and common users (the farmers engaged in animal husbandry and plantation in the subordinate institutions of farms). System administrators can be held by the persons in charge of animal husbandry in the farm, and their main function is managing animal husbandry information of farms and the limits of rights of the other users. Administrators of subsystems mainly add, delete and modify information. Common users can browse and inquire all the open information of the management system. Its diagram is shown in Fig.2.

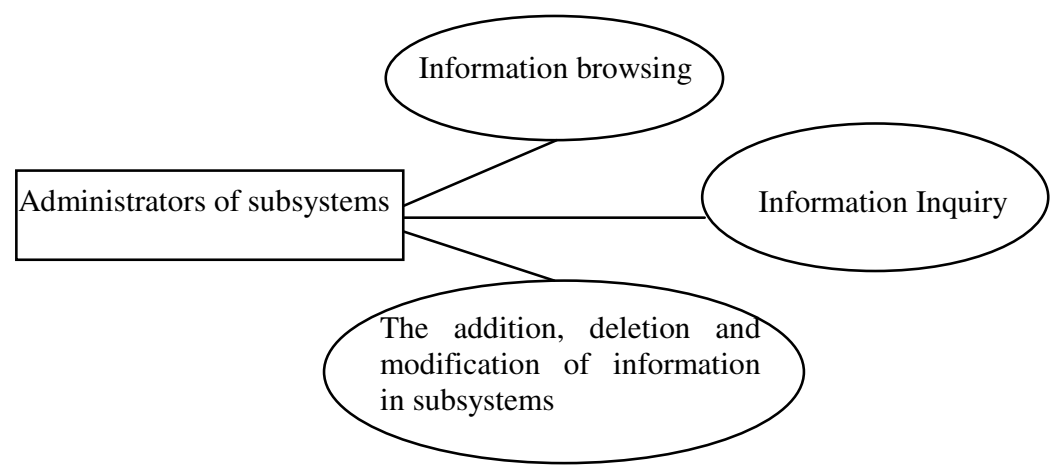

Fig. 2. Case Diagram of subsystem administrators

\section{Implementation of the System}

\subsection{The Choice of Systematic Structure}

The structural development of MIS has experienced the phases of Client/Server mode and Browser/Server mode. The traditional C/S structure adopted the open mode, but the openness is only limited to development of the system. Because it failed to provide users with the real expected open environment, the software with $\mathrm{C} / \mathrm{S}$ structure had to develop software of different versions to apply to different operating systems. In addition, its efficiency is low while the price is high. In B/S structure, users' working interface is realized though WWW browsers. The main working logic is realized in the Server, with little of it done in Browser, thus forming the so-called 3-tier structure. In this way, the load in the client is simplified, decreasing the cost and working load of system maintenance and ultimately the TCO of users (Liu Huaquan et al., 2000). 
If the system is successfully developed, the users basically need not to be trained. Their needs can be satisfied though the universal browsers and by clicking the mouse. The structure has low requirement for clients' hardware, with high degree of information resource sharing and of expansibility. And lots of users have access to wide area network. Based on these features, the system has developed and adopted B/S structure.

\subsection{The Choice of the Working Plat Form for the System}

Based on the analysis of the functions and roles of the system, many modules can be established, which are both inter-independent and interrelated. The system working platform adopted Windows 2003 Server, and background database utilized network database SQL Sever 2000 with relation model. The designing method used one of the standard database designing methods: New Orleans designing mode, which classifies designs of database into four stages: analysis of needs, conceptual design, logical design and physical design. And after the database is designed, there are two other stages: that of implementation and operation and that of operation and maintenance.

Conceptual structure design is the key to the overall database design; it refers to the process of transforming the application need got from the analysis of needs into information structure or conceptual model, and optimizing the model. The entities designed according to the above-mentioned principles include: Diary Cows Production Monthly, diary cows statistics in the end of the year, families raising more diary cows than the others, vets and technicians, facilities of animal husbandry service, information of grassland statistics, the kinds of silage and their planting methods, the plantation and harvest of silage and the plantation of silage at the end of the year and the statistics of its storage.

To guarantee the inner links between different modules and the consistency of data, in designing database, all the datasheets in the system will be managed in one database file. In the process of designing database, the fields in every datasheet have been fully optimized and assembled, so that the redundancy of the data can be reduced and the efficiency of data-utilizing can be improved.

\section{The Security of the System}

The safety management includes the safety of the operating system of the network server, the safety of the application program and the safety of database. The following aspects should be considered in designing.

\subsection{Safety Designing of Infrastructure}

(1) The back-up system: The system or the database can break down in the process of operation, and the common breakdown includes working breakdown and system breakdown. To prevent the appearance of incorrect data in the database, or to renovate the destroyed data in the database so that the database can restore to the condition of consistency, the system must be backed up regularly.

(2)To install anti-virus software and firewall to prevent virus: Installing anti-virus can monitor the operating situation of the program in the computer and can find out and wipe out the virus program, thus protecting the safety of the computer system and 
the information. To install firewall can avoid the visits of information from unauthorized users and programs.

\subsection{Safety Design of the system}

(1)Optimizing programming to avoid the illegal invasion of Web application program. Web page parameters or data shouldn't be transmitted directly, instead, they should be transmitted through component technology to achieve the purpose of conceal the parameters.

(2)The classification of the users being considered, the limits of rights should be allocated according to different users, meanwhile different operating interface should be set up to control them. To realize authority differentiation in visits, user's name and password are to be set up for subsystem administrators and their superiors, and the method of access control and identity verification should be adopted. Besides, every user should not exceed his authority to visit the contents unauthorized.

(3)To prevent the data from being accepted illegally during transmission, the system used the data encryption technology (Zhenli GU, 2005).

\section{Conclusion}

The MIS was developed according to the practical needs of the Red Star Farm in Heilongjiang province, and has its practical significance and value. The system adopted B/S structure, constructing a open comprehensive management platform of information resource for the Red Star Farm, greatly improving the efficiency and level of animal husbandry management in the farm. In designing, effective method of database technology solved the sophisticated data-processing process. The ultimate goal to develop MIS is to provide subsidiary decision-making information. The system has collected a large amount of object data in a planned way, and the data of the need for subsidiary decision-making has been abundant. Then the developing emphasis of the system would be to utilize the technologies of analysis and of data processing such as data mining technology to find out the hidden and inner valuable information, and to emphasize its analytical function of data and its roles of guiding the work and assisting decision-making, so that a more advanced decision-making and expert system can be developed.

\section{References}

Zhanying, K.: Review and Forecast about Animal husbandry of China, http: / /www. china-av.net

Huaquan, L., et al.: The Development of 3-tiers based B/S structure database management system. Microcomputer \& Its Applications 19(3), 43-45 (2000)

$\mathrm{Gu}, \mathrm{Z}$.: Analysis and Implementation of SQL Server Database Technology for Remote Access. Computer and Modernization 8, 56-58 (2005)

Songtao, Z.: Development of Dynamic web site based ASP. Publishing House of Electronics Industry, Beijing (2006)

Ruijun, G., et al.: Examples of Database developed. Publishing House of Electronics Industry, Beijing (2005) 